\title{
Restoration of Endodontically Treated Molars: Lights and Shadows of the Endocrown Approach
}

\author{
Rihab D*, Zohra N and Mounir C \\ Department of Fixed Prosthodontics, Faculty of Dental Medicine, Monastir University, \\ Tunisia
}

*Corresponding author: Rihab Dakhli, Assistant Professor, Department of Fixed Prosthodontics, Research Laboratory of Occlusodontics and Ceramic Prostheses, Faculty of Dental Medicine, University of Monastir, Monastir ,Tunisia, Email: rihab1983@gmail.com

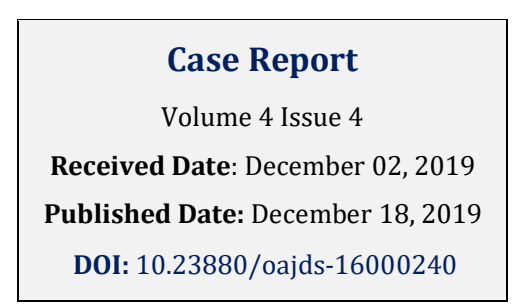

Case Report

Volume 4 Issue 4

Received Date: December 02, 2019

DOI: $10.23880 /$ oajds- 16000240

\section{Abstract}

The aim of the restoration of extensively damaged endodontically treated teeth is ensuring teeth long-term by enhancing their biomechanical behavior deteriorated. This procedure remains a clinical challenge and a common problem encountered by dentists since the retention and the resistance still usually compromised. Thus crowns supported by post and core were required for the restoration of such teeth. Currently, with the revolution of adhesive techniques, the advent of the concept of minimally invasive dentistry as gold standard had turned upside down this concept. So endocrowns was considered as a reliable alternative esthetic and conservative facing such situations. Such ceramic restoration is monolithic and is anchored in the pulp chamber, taking benefit the micromechanical retention properties of the pulp-chamber walls and micromechanical retention of bonding.

Keywords: Endocrown; Minimally invasive; Bonding; Esthetics; Ceramics

Abbreviations: ETT: Endodontically Treated Teeth; CAD-CAM: Computer-Aided Design and Computer-Aided Manufacturing; ETT: Endodontically Treated Teeth; RCT: Root Canal Treatment.

\section{Introduction}

For many years, the restoration of endodontically treated teeth (ETT) with extensive coronal destruction has been a challenging procedure in restorative dentistry. The gold standard has been the manufacture of metalceramic or all ceramic full coverage crowns, with or without the presence of post [1]. However, placing a post though contributing to the retention of the core portion of the restoration, it may cause accidental perforations and weakening the root [2]. Moreover and over the years, the importance of the preservation of healthy tooth structure with advances in adhesive dentistry, computer-aided design and computer-aided manufacturing (CAD-CAM) technologies, and the advent of reinforced-ceramic materials have resulted in the introduction of new concepts regarding the restoration of teeth avoiding, thus, aggressive macromechanical approaches.

One of this new alternative treatment modality include the endocrown restoration, which reduce the risk of failure when preparing intracanal post [1,3]. Endocrowns, defined as "bonded overlay restorations", has been 
introduced for the first time in 1995 by Pissis. They are anchored to the pulp chamber and on the cavity margins in order to increase the machromechanical retention of the crown whereas micromechanical retention is assured by adhesive bonding to the remaining tooth structure [4]. The purpose of this present paper is to describe the clinical characteristics of the preparation design of endocrown restoration through different clinical cases. Then, we will discuss the indication and the advantages of this conservative approach.

\section{Case Presentation}

A 24-year-old female patient, student in dental medicine attended the prosthodontic department, after performing and finishing the endodontic treatment of the first right mandibular molar (teeth \#46). She suffered from chronic periodontitis, but the situation was stable after the therapy. She was very aesthetically demanding. The medical history was non-contributory.

Radiographic and clinical examinations were performed. At the first appointment, a very wide access cavity was notified, and a crack in the mesial part of tooth above a cavity that opens into the mesial cervical embrasure and very thin remaining walls were detected (Figure 1). The radiographic findings revealed well-sealed canals without no changes in periapical area (Figure 2).

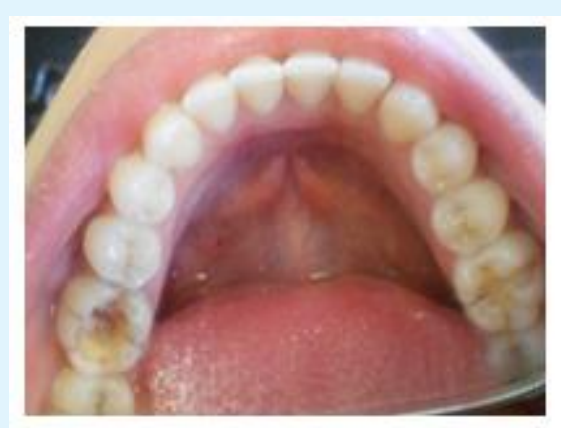

Figure 1: Clinical condition of tooth \#46 with large access cavity and the crack of mesial wall.

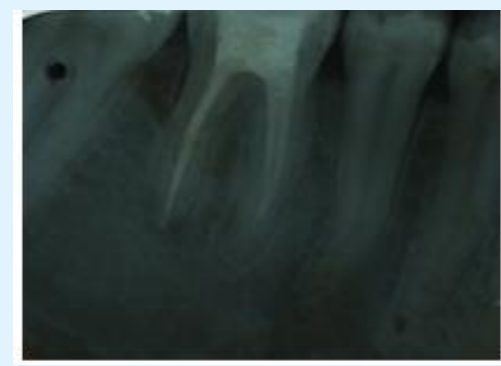

Figure 2: Periapical radiographic.
The patient had an acceptable oral hygiene and a favorable occlusion. The working models were obtained by taking impressions of the mandibular and maxillary arches, using irreversible hydrocolloid (CA 37, Cavex, Holland).

After discussing the treatment options and since the patient was well informed, it was decided to restore the tooth with an endocrown manufactured from lithium disilicate ceramic (IPS e.Max CAD) thanks to its superior mechanical and optical properties. Before any dehydration of the teeth, the selection of the color of ceramic block led to an A2 shade restoration using a digital shade guide.

Once the provisional restoration was removed, endocrown's preparation, which required specific guidelines, was initiated. The Occlusal reduction was about 2 to $3 \mathrm{~mm}$; until reaching the mesial cavity; using a diamond wheel bur parallel to the occlusal plane. The aim of this reduction was to achieve a flat surface. The cervical margin simulating a butt join was prepared in supragingival level, but no ferrule was created (Figure 3). Differences in levels between various parts of the cervical margin must not exceed $60^{\circ}$ to escape a staircase effect. Then we moved for the preparation of the second key part of endocrown's success: the central retentive cavity. With a cylindrical-conical diamond bur orientated along the long axis of the tooth, the central retentive cavity of at least $3 \mathrm{~mm}$ depth was prepared which extended into the pulp chamber space with occlusal divergence of 8 to 10 degree as well as all internal line angles were rounded and smoothened.

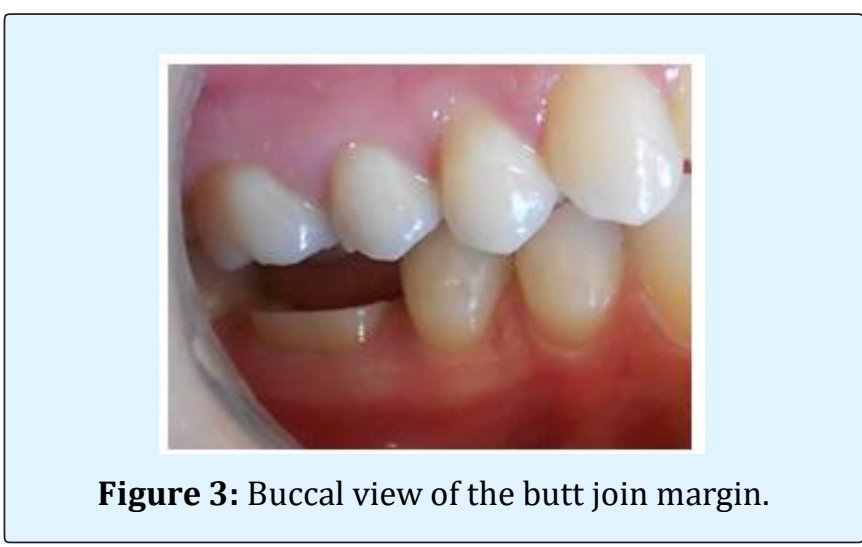

The pulpal floor was carefully untouched but the entrance of the pulpal canal was slightly opened in a way to preserve the saddle-like anatomy of the pulp chamber which is too advantageous for the success of the restoration. Provisional restoration was fabricated by the 
injection of the provisional restorative material $(3 \mathrm{M}$, Protemp $^{\mathrm{TM}}$ Plus temporization material shade $\mathrm{A} 2$ refill, $3 \mathrm{M}$ United Sates) in the silicone plates obtained from the diagnostic wax-up. After finishing and polishing, the restoration was cemented (Figure 4) with eugenol-free temporary cement (NE Temp-Bond, Kerr, Italy).

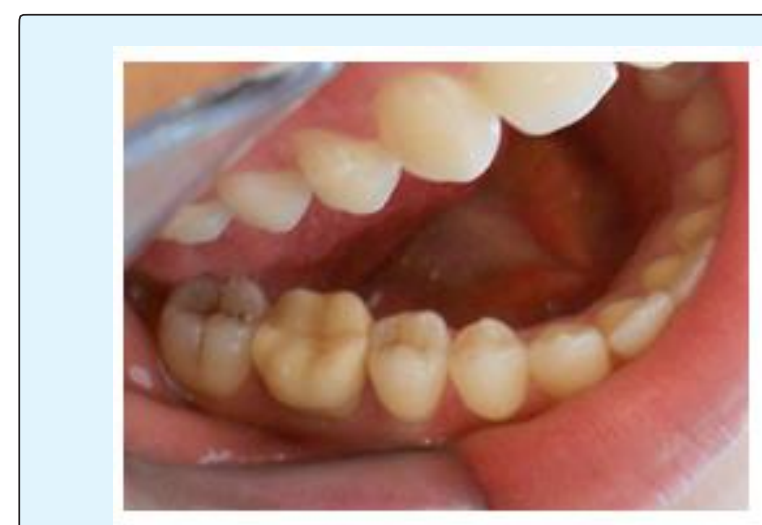

Figure 4: Occlusal view of the provisional restoration.

After 5 days, provisional restoration was removed and preparation perfectly cleaned. Once we checked the interocclusal space and if there were any irregularities or undercuts in the pulp chamber, the impression was taken using additional silicone (Elite HD+, Zhermack, Italy) and sent to the laboratory. All manufacturing steps using CADCAM technology were done starting by scanning the model, then the virtual restoration was designed and the final restoration was milled according to the corresponding color matching (Figure 5). Before cementation, the finished endocrown was checked for shade, marginal integrity, fit and occlusion in the patient's mouth then sent back for color adjustments and application of glaze (Figure 6).

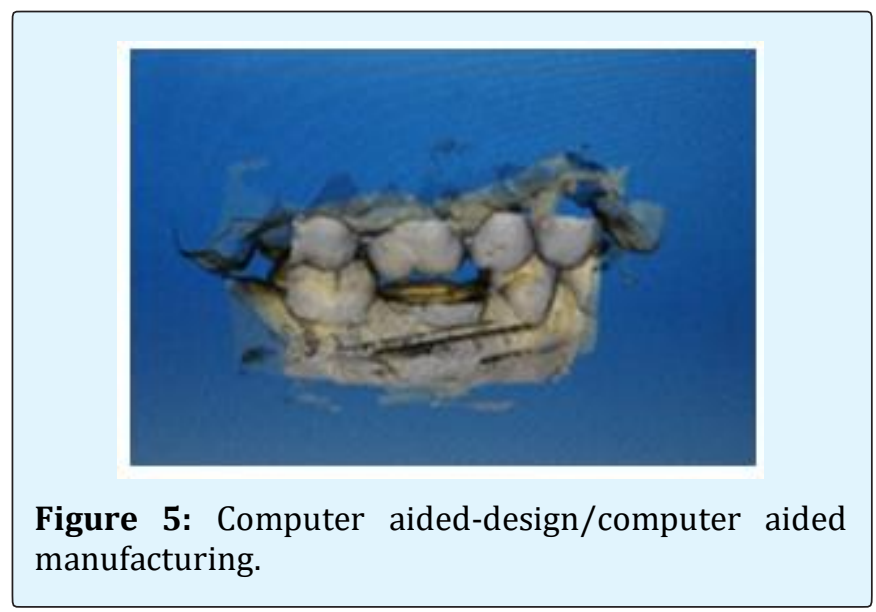

Rihab D, et al. Restoration of Endodontically Treated Molars: Lights and Shadows of the Endocrown Approach. J Dental Sci 2019, 4(4): 000240.

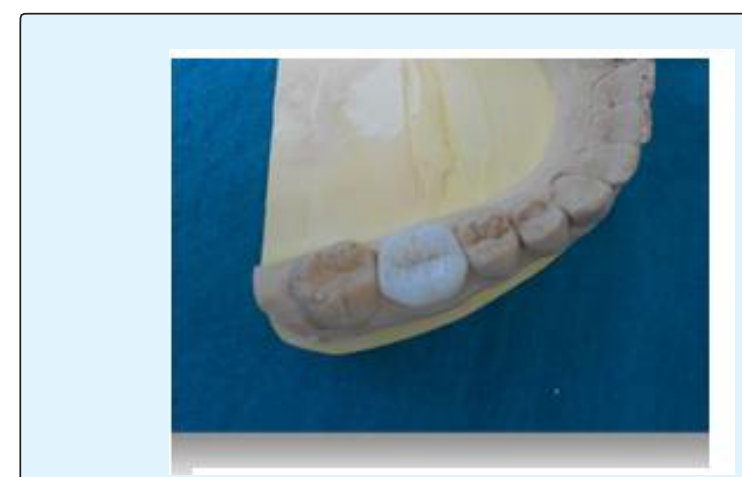

Figure 6: Fitting the restoration on the model.

A total etch dual cure resin (Variolink II, Ivoclar/Vivadent, Liechtenstein) was used. Bonding procedures were carried out starting with the treatment of the restoration: the inner face of the glassy endocrown was etched with hydrofluoric acid for 20s (Dentobond Porcelain Fix, Itena, France) followed by rinsing with distilled water, dried and coated with silane application, thinning with a gentle air stream for 60 seconds (Figures 7 \& 8).

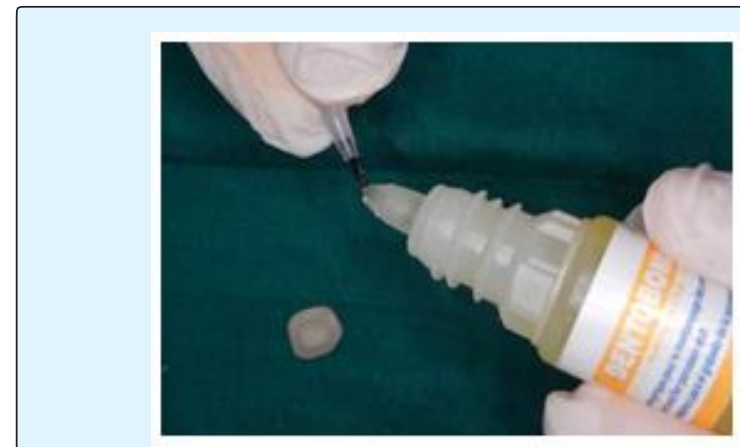

Figure 7: Application of the hydrofluoric acid to etch the inner surface then applying a silane.

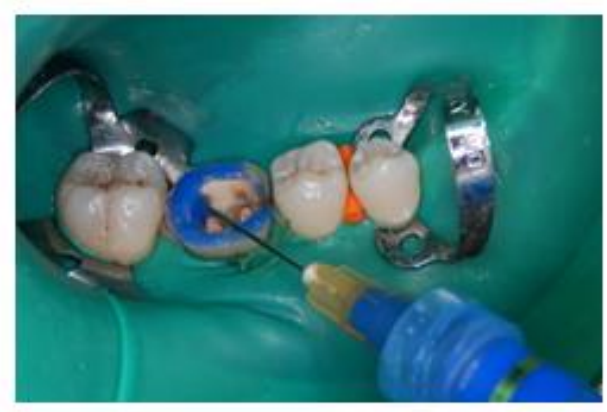

Figure 8: Treatment of the inner surface of the tooth after protection with a Rubber Dam: Etching with orthophosphoric acid then applying a thin layer of adhesive. 


\section{Open Access Journal of Dental Sciences}

An initial polymerization for $5 \mathrm{sec}$ was carried to make the cement excesses' removal easier, and then completed by a polymerization for $60 \mathrm{sec}$ on all surfaces. No occlusal adjustment was done in our case (Figure 9) and the restoration was characterized acceptable even after 4 years follow-up periods.

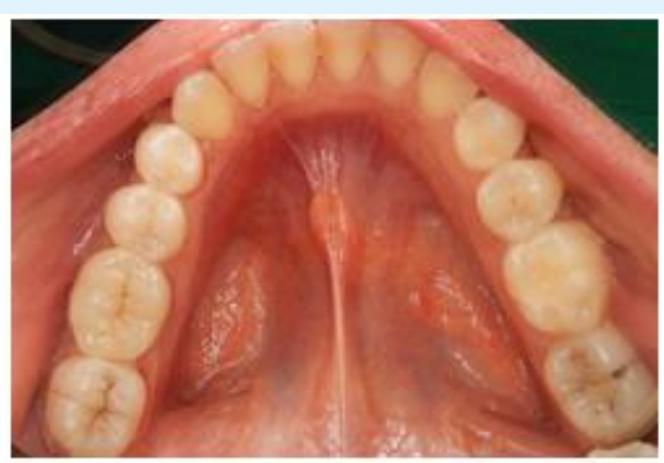

Figure 9: Final restoration after final polymerization and checking occlusal adjustment.

\section{Discussion}

Tooth rehabilitation may depend on several factors, including the intake and the limitations of the restorative technique, the amount of residual tissue, either the tooth is endodontically treated or not and the patient's wishes and expectations [5]. Restoring endodontically treated teeth (ETT) still a challenging procedure. In fact, these teeth are prone to fracture after root canal treatment (RCT) which is vulnerable to failure and contamination in the absence of post-endodontic restoration. That is why, it is very important to restore the teeth in a very short time after RCT [6].

For several years, the rehabilitation of non-vital teeth with extensive coronal destruction has been performed by combining metal posts with core materials, then the placement of full coverage ceramic crowns. A part from the fact that is invasive regarding remaining sound coronal tissue, such a procedure include the risk of root perforation during preparation and weakening the toothroot-complex. Full coverage crown preparations lead to greater tooth wear, clinical time-consuming, and costs to expensive [7].

So thanks to the advances in adhesive technologies and materials, endocrowns were suggested as an alternative to post and core retained ones more conservative for the restoration of posterior teeth [8].
They have been recommended for teeth where post and core restorations become impossible in the presence of short, calcified and curved root canals, short, or curved root canals and especially with short clinical crowns [9].

The first endocrown report known as "the monoblock porcelain technique" was made by Pissis in 1995. However, in 1999, the term "endocrown" was used from Bindl \& Mörmann [10] to describe a ceramic crown with extension into the pulp chamber.

Combining the crown and the core as a single unit, the retention is assured through both macro- and micromechanical means, making it a suitable approach for teeth with a low occluso-gingival distance that make retention insufficient for a full crown [6,11]. Furthermore, this restoration have several benefits as the provision of an endocrown is a relatively easy, cost-effective procedure that requires less chairside time, less stress concentration because of the reduction in the non-homogenous material present without forgetting especially the minimal invasive design. Moreover, with supragingival margins facilitate plaque control should be well facilitated $[8,12]$.

According to recent systematic review and metaanalysis, Sedrez reported that endocrown restorations our performed conventional restorations by means of fracture strength outcome [13]. And in the short, medium, and long term excellent survival rates have been reported when restoring molars in this way [14]. Finite element analysis from in vitro studies approve and confirm this idea from the moment that they could withstand physiological chewing forces without fracture or debonding. They were more resistant to failure than those treated with glass fiber reinforced composite posts [9].

In fact, besides problems related to the choice of the quality and the quantity of cement, the polymerization, relationship with the root canal length, and the cavity configuration factor, there is also the possibility of the presence of air bubbles forming during the insertion of the cement and placement of the glass fiber post which can lead to bonding failure [15].

In addition, it was demonstrated that catastrophic failures by means of root fractures were about $6 \%$ for endocrowns against 29\% for crowns and loosening (71\%) was reported as the most failure [14]. Such monolithic restoration can be milled using either the heat-pressed technique or the newly one "CAD/CAM". The latter provides esthetics, the speed of the process as long as it will be fabricated in a single block thus the best accuracy. Thus, it may reduce human errors and control all the 


\section{Open Access Journal of Dental Sciences}

variables to decrease the margin of errors $[1,16]$. Nevertheless, Menezes preferred using ceramic injection by the lost wax technique as the best option when the walls of the preparation were very thin [15].

To be successful, some guidelines and rules should be respected and followed for an endocrown restoration. A cervical margin by mean of butt join of at least $2 \mathrm{~mm}$ wide by $60 \%$ minimum of the dental circumference is suggested preferably placed supragingival and a pulpal chamber of at least $3 \mathrm{~mm}$ of depth is recommended. A divergence in the occlusal wall of $5-7^{\circ}$ is necessary for two reasons: it is the nearest value favoring stability and retention and the minimum recommended for taking intraoral scanner with Cerec camera [17].

Sometimes, modifications can be made for functional and esthetic reasons such as the presence or not of a uniform ferrule [1]. Besides, it is against minimally invasive dentistry, Taha $D$, et al. in their studies demonstrated that endocrowns with ferrule design had higher values concerning fracture resistance than endocrowns with butt margin design with significant difference statistically It has the ability to increase the dentin surface available for adhesion too [18].

Elalem IA, et al. showed on their study that the design and the width of the finish line affect the amount of marginal distortion during firing. That is why the butt joint margin showed higher marginal gap than the deep chamfer finish line. Nevertheless, both were within the clinical acceptable range regarding marginal and internal fit [16].

As far as regarding the pulpal chamber, since the occlusal reduction is inversely proportional to the depth, studies findings revealed that endocrowns with occlusal thickness about $3.5 \mathrm{~mm}$ had higher fracture resistance mean values than those with occlusal thickness about 2 $\mathrm{mm}$ however no significant difference was noted statistically $[13,18]$. This pulpal chamber thanks to its trapezoidal shape for the lower molars and triangular for the upper ones ensures the anti-rotational blockage of the restoration and enhance its stability [17]. Therefore, the higher is the depth of pulp chamber, the higher is the bonding surface available and thus the risks of displacement is limited. That emphasizes to be the more conservative regarding dental remnant amount. In fact, the higher the amount of residual tooth tissue, the lower the stress concentration in the cement line $[7,14]$. Nevertheless, intracanal extension of the endocrown preparation should be avoided as it affect negatively both the marginal adaptation and the internal fit of the final restoration, and its clinical performance [19].

All materials able to be acid etched and resin bonded to tooth tissue are recommended for endocrown restoration as the retention depends mainly on bonding. Thus, lithium disilicate glass-ceramics, hybrid nanoceramics, fiber-composites and zirconia-reinforced lithium silicate glass-ceramic are all of them materials that could be milled for the fabrication of endocrown although their microstructure and physical properties are different [3]. Reinforced glass ceramic with leucite or lithium disilicate are considered as the best option thanks to the higher flexural strength they exhibit and their resistance against the occlusal forces [1]. However, the contraction (15-20\%) of the material that occurs during the second sintering process could be too disadvantageous. Indeed, debonding is the main reason for failure in endocrown restorations [6,7]. Sedrez JA, et al. had demonstrated that E.max endocrowns compared to those fabricated from conventional composite, bulk fill composite and conventional composite using resin adhesives; appeared to present more aggressive failures, and support weakly stress [5]. In the other hand, Zirconiareinforced lithium silicate glass ceramic (VITA SUPRINITY, VITA Zahnfabrik, Bad Sackingen, Germany) had superior properties than disilicate glass ceramics by means of flexural strength, fracture toughness, hardness and elastic modulus [6].

Recently, polyetheretherketone (PEEK), a good performed polymer, has been introduced in dentistry. By the introduction of a modified PEEK material embedded with 20\% ceramic fillers (BioHPP; Bredent $\mathrm{GmbH}$ ); endocrown's restoration of ETT using PEEK seems to be very viable and interesting. Its major advantage the modulus of elasticity about 4-GPa allowing him to dampen force transmission to the restoration as well as the tooth root besides its ability to be a good adherent to dental tissue [11]. Another material which had approved its interest and superiority than feldspathic porcelain or lithium disilicate by means of fracture resistance was recommended for the fabrication of endocrowns is the resin nanoceramic. Nevertheless, it seemed to reveal more microleakage [20].

The combination of the two polymerization mechanisms, physical and chemical, guarantee polymerization of the restoration even in the absence of access to light. Therefore, in case of endocrown our choice to use dual cement was justified by the fact that the lightcuring unit light needed to pass through the ceramic thickness. Moreover, the interest of initial polymerization 


\section{Open Access Journal of Dental Sciences}

of the cement allowed the removal of excesses more easily and avoided such a trauma to the marginal gingival tissue if mechanical withdrawal of overhanging cement are needed [15].

Finally, occlusal adjustments are often necessary and may lead to damaging the ceramic or removing the restoration' characterization. Thus, it is important to succeed the fit and avoid any adjustments so that the occlusal anatomy remain stable. In this context, the biogeneric option in CAD/CAM, offer real advantages thereby avoiding the use of diagnostic waxing and providing virtual restoration that better fits and adapts to the scanned preparation and antagonist anatomy [21].

For incisors and premolars, the results concerning the choice of endocrowns as a treatment option are still not concluded for incisors. Whereas, premolar endocrowns showed similarity with full coverage crowns with postretained restorations regarding fracture resistance, survival rates and stress distribution [14].

\section{Conclusion}

When it is about restoring endodontically treated teeth; the main objective is ensuring and enhancing restoration long-term prognosis with minimally invasive approach. Endocrown as a monolithic restoration, whose anchorage occurs in the pulp chamber, was considered as a viable alternative judged more than satisfactory regarding clinical surviving. For the success and longevity of this restoration, such guidelines must be followed and respected such the selection of cases, the adequate preparation, the choice of material and the mastery the bonding.

Acknowledgment: The author would like to thank Fathi Troudi dental technician for the laboratory work.

\section{References}

1. Konstantinos T, Maria T, Paris G, Effrosyni T (2018) Endocrown restorations for extensively damaged posterior teeth: clinical performance of three cases. Restor Dent Endod 43(4): e38.

2. Taha D, Sebastian S, Ahmed S, Marwa W, Tarek S (2018) Assessment of marginal adaptation and fracture resistance of endocrown restorations utilizing different machinable blocks subjected to thermomechanical aging. J Esthet Restor Dent 30(4): 319-328.
3. Wiam A El Ghoul, Mutlu O, Hani O, Hani T, Ziad S (2019) Effect of different CAD-CAM materials on the marginal and internal adaptation of endocrown restorations: An in vitro study. J Prosthet Dent 3913(18): 31097.

4. Laden Gulec, Nuran Ulusoy (2017) Effect of Endocrown Restorations with different CAD/CAM Materials: 3D Finite Element and Weibull Analyses. BioMed Research International 2017: 1-10.

5. José Augusto SP, Eliseu AM, Lisia LV, Maximiliano SC, Tatiana PC (2019) New material perspective for endocrown restorations: effects on mechanical performance and fracture behavior. Braz Oral Res 33: $\mathrm{e} 012$.

6. Jieli Sun, Wenhao Ruan, Jiahui He, Xiaoyu Lin, Bowen $\mathrm{Ci}$, et al. (2019) Clinical efficacy of different marginal forms of endocrowns: study protocol for a randomized controlled trial. Clinical Trials 20: 454.

7. Tribst JPM, Dal Piva AMO, Madruga CFL, Valera MC, Borges ALS, et al. (2018) Endocrown restorations: Influence of dental remnant and restorative material on stress distribution. Dent Mater 34(10): 1466-1473.

8. Gresnigt MM, Özcan M, van den Houten ML, Schipper L, Cune MS (2016) Fracture strength, failure type and Weibull Characteristics of lithium Disilicate and multiphase resin composite endocrowns under axial and lateral forces. Dent Mater 32(5): 607-614.

9. Biacchi GR, Basting RT (2012) Comparison of fracture strength of endocrwns and glass fiber post-retained conventional crowns. Oper Dent 37(2): 130-136.

10. Bindl A, Richter B, Mörmann WH (2005) Survival of ceramic computer-aided design/manufacturing crowns bonded to preparations with reduced macroretention geometry. Int J Prosthodont 18(3): 219-224.

11. Panagiotis Z, Eleonora B, Gregory P (2017) Using modified polyetheretherketone (PEEK) as an alternative material for endocrown restorations: A short-term clinical report. J Prosthet Dent 117(3): 335-339.

12. Roopak Bose C, Mohan Thomas N, Shamina P, Roshni S, Shiny B, et al. (2013) Restoration of endodontically treated molars using all ceramic endocrowns. Case Reports in Dentistry 2013: 210763. 


\section{Open Access Journal of Dental Sciences}

13. Sedrez-Porto JA, Rosa WL, da Silva AF, Münchow EA, Pereira-Cenci T (2016) Endocrown restorations: A systematic review and meta-analysis. J Dent 52: 8-14.

14. Nicolas G, Mathieu C (2019) Endocrowns: A systematic review. The Journal of Prosthetic Dentistry.

15. Rafael MS, Carlos AVE, Maria TA, Maria FLN, Sérgio KI, et al. (2016) Endocrown: a conservative approach. Brazilian Dental Science 19(2).

16. Inas AE, Rabab MI, Ahmed MH (2019) Clinical Evaluation of the Marginal Integrity, and Internal Fit of E-Max Endocrown Restorations with Different Marginal Preparation Designs. Ex-Vivo Study. Journal of Dental and Oral Health 1:128.

17. Fages M, Bennasar B, Raynal J, Margerit J (2013) The Endocrown: A different type of All-Ceramic Reconstruction for Molars. J Can Dent Assoc 79: 140.
18. Taha D, Sebastian S, Christine S, Ahmed S, Marwa W, et al. (2017) Fracture resistance and failure modes of polymer infiltrated ceramic endocrown restorations with variations in margin design and occlusal thickness. J Prosthdont Res 62(3): 293-297.

19. Gaintantzopoulou M, El-Damanhoury H (2016) Effect of Preparation Depth on the Marginal and Internal Adaptation of Computer-aided Design/Computerassisted Manufacture Endocrowns. Oper Dent 41(6): 607-616.

20. El Damanhoury H, Haj-Ali R, Platt JA (2015) Fracture resistance and microleakage of endocrowns utilizing three CAD-CAM blocks. Oper Dent 40(2): 201-210.

21. Da Cunha LF, Gonzaga CC, Pissaia JF, Correr GM (2017) Lithium silicate endocrown fabricated with a CAD-CAM system: A functional and esthetic protocol. J Prosthet Dent 118(2): 131-134. 